•综述・

\title{
中国巨蚓科蚯蚓的起源与演化
}

\author{
蒋际宝 $^{1,2,3}$ 邱江平 $1,2,3 *$ \\ 1 (上海交通大学农业与生物学院, 上海 200240) \\ 2 (农业部都市农业重点实验室, 上海 200240) \\ 3 (国家林业局上海城市森林生态系统国家定位观测研究站, 上海 200240)
}

摘要: 截至2017年中国已记录巨蚛科虾蚓579种(亚种), 优势类群为远盲蚫属(Amynthas)与腔蚂属(Metaphire), 其 丰富的物种多样性值得深入研究。近年来的研究指出联合形态分类与分子系统发育可较好地探讨中国巨䗁科蚯蚂 的起源、分化与扩散。本文概述了中国巨蚛科蝶蚛物种的起源、分化时间以及扩散历程，探讨了受精囊孔、盲肠 形状等重要特征的演化, 分析了现行分类系统的缺陷。多项研究表明, 中国巨䳋科主要类群的祖先起源于中南半 岛, 于白严纪末期至新生代初期进入中国, 在新生代得以繁荣发展; 受精囊孔的对数或位置是多起源的, 盲肠形 状演化中有祖征重现的现象, 故使用少数形态特征进行类群划分的现行分类系统有待改进。此外, 中国巨蚂科蚟 蚂的具体演化机制及影响因素仍不明确。因此, 在未来研究中整合形态特征、分子数据与地理格局、地质历史及 环境因子等信息, 定量分析类群演化与古地理、生物与非生物因素间的关系, 将有助于全面厘清中国巨蚂科虾蚂 演化的具体机制。

关键词：巨蚂科; 蚯蚂; 起源; 物种分化; 特征演化

\section{Origin and evolution of earthworms belonging to the family Megas- colecidae in China}

\author{
Jibao Jiang ${ }^{1,2,3}$, Jiangping Qiu ${ }^{1,2,3^{*}}$ \\ 1 School of Agriculture and Biology, Shanghai Jiao Tong University, Shanghai 200240 \\ 2 Key Laboratory of Urban Agriculture, Ministry of Agriculture, Shanghai 200240 \\ 3 Shanghai Urban Forest Research Station, State Forestry Administration, Shanghai 200240
}

\begin{abstract}
A total of 579 Megascolecidae species have been reported in China through 2017. Most belong to the genera Amynthas and Metaphire. The family's rich diversity merits further investigation into its evolutionary history. The arc of research indicates that analysis based on taxonomical and molecular methods reveals the evolutionary history of the family Megascolecidae in China. This paper summarizes updated findings regarding origin, speciation and dispersal of Megascolecidae in China, and discusses the evolution of major familial characteristics (e. g. spermathecal pores, caeca). Several analyses suggest that the ancestors of Megascolecidae in China may have come from the Indo-China Peninsula. Speciation radiation likely occurred after the Cretaceous-Palaeogene mass extinction, and species richness increased rapidly during the Cenozoic period. Ancestral range reconstruction analysis shows that species-groups are polyphyletic and that evolutionary reversal often resulted in sharp evolution of caeca, so we suggest that the current taxonomic system of Megascolecidae, which is based on a few morphological characters, should be reconstructed. We also note that the specific mechanism of evolution in Megascolecidae has not been studied. Hence, future research to reveal the specific evolutionary mechanism of Megascolecidae earthworms requires more systematic sampling of this family, combined with morphological research, phylogeny construction and analysis of geographical patterns, geological history and environmental factors.
\end{abstract}

Key words: Megascolecidae; earthworm; origin; speciation; evolution of characteristics

收稿日期: 2018-04-10; 接受日期: 2018-10-02

基金项目: 国家自然科学基金(41471204, 31401947)和博士后科学基金(2017M611546)

* 通讯作者 Author for correspondence. E-mail: jpq@sjtu.edu.cn 
蚯蚓是最重要的土壤动物类群之一, 几乎见于 世界各地所有温湿度合适的土壤环境中, 在生态系 统中占有非常重要的地位(邱江平, 1999)。它们在土 壤结构的形成、促进土肥相融、提高植物营养、改 善土壤通透性、提高储水与保肥能力等方面起着十 分关键的作用(邱江平, 2000); 也对陆地生态系统 本身具有深刻的影响, 可以促进土壤有机质的分解 和转化, 调节陆地碳汇(Zhang et al, 2013)。

目前世界上已发现的蚯蚓超过6,000种，去除 同物异名的物种后实际可达3,000-3,500种(Csuzdi, 2012)。在分类学上, 它们归属于寡毛纲(Oligochaeta) 的单向蚓目 (Haplotaxida) 和正蚓目 (Lumbricida), 包 括18个科, 其中巨蚓科(Megascolecidae)、正蚓科 (Lumbricidae) 和真蚓科(Eudrilidae) 是陆生蝶蚓中发 展最广的类群, 也是已知的最大类群 (邱江平, 1999)。巨蚓科记载的物种数在 1,000 种以上, 主要分 布在中国、东南亚各国、日本、韩国、朝鲜、澳大 利亚及新西兰等地, 部分物种广泛分布于世界各 地。正蚓科和真蚓科已知的物种也都在 500 种以上, 前者主要分布在欧洲和北美, 后者多见于撒哈拉沙 漠以南的热带非洲地区。

迄今为止, 中国共记录有蚯蚂9科28属640种 (黄健等, 2006; 蒋际宝, 2016; Shen et al, 2016; Zhao et al, 2017), 是亚洲乃至全球报道虾蚓物种数最为 丰富的国家之一。这些物种主要属于巨蚓科、正蚓 科、链胃蚓科(Moniligastridae)等, 其中巨蚓科作为 最大的优势蝤蚓类群, 占中国蚯蚓物种总数的 $90 \%$ 以上, 共记录有579种(亚种)(黄健等, 2006; 孙静, 2013; 赵琦, 2015; 蒋际宝, 2016)。该科的远盲蚓属 (Amynthas)与腔蚓属(Metaphire)为优势类群, 其中 远盲蚓属有443种(亚种), 腔蚓属有126种(亚种), 合 计占物种总数的 $88.9 \%$; 其他属的物种较为稀少: 巨蚓属(Megascolex) 1种, 环棘蚓属(Perionyx) 1种, 近盲蚓属(Pithemera) 3种, 扁环蚓属(Planapheretima) 4种, 多环蚓属(Polypheretima) 1种, 且近盲蚓 属与多环蚛属仅在台湾地区有记录(黄健等, 2006)。 从已统计的资料可知, 我国巨蚓科物种大多分布于 温湿条件较好的南方诸省(图1), 广西、海南、台湾 与四川等省份各记录有79种以上, 而整个北方地区 仅记录有 13 种, 且多数是适应能力较强的广布物 种。这既是物种分布格局不均匀造成的(孙静, 2013), 也与北方分类研究投入的采样工作较少有关(蒋际宝,

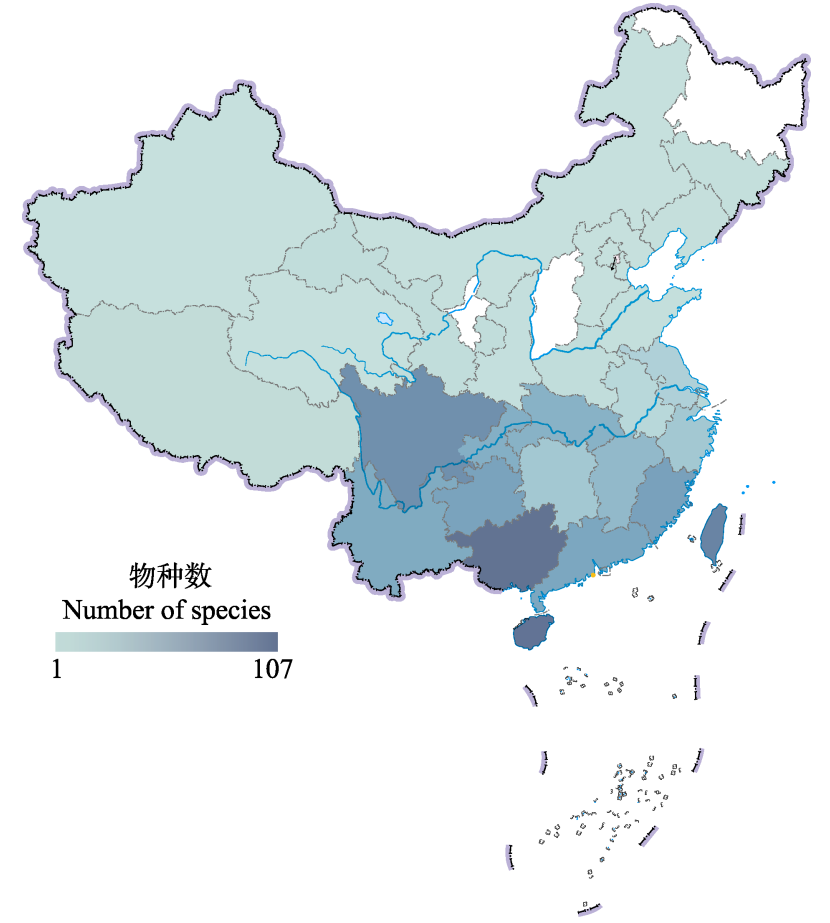

图1 在 2017 年前已调查的中国巨蚓科蚯蚓物种分布示意 图。白色代表调查空白省份。

Fig. 1 Distribution diagram of investigated Megascolecidae species in China before 2017. Some provinces with no surveys are blank.

2016)。

中国巨蚓科蚯蚓丰富物种多样性的成因及其 扩散历程均值得深入探讨。分类学者最早是通过形 态特征及地理分布特点来初步探讨其起源与演化。 20世纪初, Michaelsen (1929a, b, 1931)基于分类研 究推测中国巨蚂科可能发源于菲律宾或婆罗洲, 再 扩散出去, 东到太平洋波罗的群岛, 南达澳洲北部 海角, 西至安达曼群岛; 印度的巨蚓科物种很少, 而中国和日本的物种多样性非常丰富。陈义(1956) 也指出巨蚓科在印度极少, 而在中国是优势类群, 其祖先可能从南洋及菲律宾等地扩散至中国大陆, 而后发展出多样的物种。针对上述推断, 近年来多 位学者已针对中国巨蚂科形态分类与分子系统发 育开展了大量工作, 深入探讨了主要类群的起源 与演化, 并取得一系列重要的研究成果。本文将通 过文献汇总与比较, 总结出中国巨蚂科虾蚓的起 源、分化与扩散规律, 并阐述其主要形态特征的演 化过程, 从而有助于更深入理解中国蚳蚓的进化 历程。 


\section{中国巨蚂科的起源、分化与扩}

近一个世纪以来, 全球虾蚓科以上的演化研究 已经取得了重要成果。在Michaelsen (1900，1921, 1929a, b)工作的基础上, Gates (1972)、Bouché (1972, 1998)、Jamieson (1988)、Qiu和Bouché (1998)、Omodeo (1998)等对蚯蚓科以上阶元的分类、分布、系统 发育及其与古地理变迁之间的关系开展了深入研 究, 探讨了各个类群的起源与演化历程。Qiu和 Bouché (1998)建立了新的分类系统, 设置了链胃蚓 超科(Moniligastroidea)、正蚓超科(Lumbricoidea)、 沙蚓超科(Glossoscolecoidea)、巨蚓超科(Megascolecidea)等。他们推测, 蚯蚓起源于冈瓦纳古陆(Gondwana), 在泛古陆 (Pangea) 时期传播到劳亚古陆 (Laurasia) (即今天的北美大陆和欧亚大陆欧洲部分) 的蚯蚓祖先演化形成了正蚓超科; 在泛古陆解体以 后，冈瓦纳古陆分化、漂移形成了今天的南美大陆、 澳洲大陆、欧亚大陆的东南部和非洲大陆等板块, 虹蚓也随之演化形成了亚洲、非洲与澳大利亚的巨 蚓超科和链胃蚓超科为主的区系, 以及南美大陆沙 蚓超科为主的区系(附录1，2) (Bouché, 1983，1998; Qiu \& Bouché, 1998)。从他们的分析可知, 印度次大 陆在白严纪早期 (约 $120 \mathrm{Ma}$ ) 以棘蚓科 (Acanthodrilidae)为主, 在始新世中期(约50 Ma)撞上亚洲大 陆后，有部分巨蚂科物种逐步迁徙于该地，造成次 大陆上有了巨蚓科虾蚓, 但是物种数量较少。

Bouché (1998)通过对蚯蚓的多个重要形态特 征(雄孔、精巢与砂囊等)进行分析, 系统论述了各超 科的形态结构与功能关系及特征的演化意义, 并结 合类群地理分布特点及板块地质变迁的重要历史 事件推测了各个虾蚓超科的分化时间: 正蚓超科与 巨蚓超科大约在三叠纪中后期即约 220 百万年前 (Ma)发生分化, 巨蚓超科中的巨蚓科起源时间可能 介于 110 百万年前与 220 百万年前之间(附录3)。由于 蝶蚓研究中无化石数据可用于类群分化时间校正, 因此需要依靠分子系统发育研究直接对类群的起 源、分化与扩散等历史演化过程进行进一步的验证 与探讨。譬如, Domínguez等(2015)通过分子钟研究 验证了正蚓超科与巨蚓超科、沙蚓超科等分化时间 在三叠纪后期(约201百万年), 正蚓超科内部的分化 起源于白严纪早期(约125.2百万年) (Domínguez et al, 2015)。现阶段, 除正蚓科外(Qiu \& Bouché, 1998;
Domínguez et al, 2015), 对其他科及以下阶元的起 源与演化认识还很不足。而中国的巨蚓科䗒蚓的起 源、分化与扩散又是怎样的呢? 以上这些正蚓超科 的研究可为巨蚓科在全球的地理分布格局及历史变 迁及后续类群的溯祖分析、分子钟估计等提供参考。

基于多个线粒体或核基因联合开展生物信息 学分析, 已成为虾蚓分子进化研究的重要手段。近 年来，孙静(2013)对我国巨蚓科优势类群远盲蚓属 117 种蚯蚓的 5 个线粒体基因 (COI、COII、ND1、12S 与16S)与核基因28S开展的分子系统发育研究发现, 我国远盲蚓属分化为数个地域特征明显的单系群, 并推测这些类群的祖先起源于中南半岛。

随后，蒋际宝(2016)使用321个物种587个样本 5 个线粒体基因联合构建了中国巨蚓科分子系统发 育关系，并在该基础上采用非相关对数正态松散分 子钟及估计的碱基替代速率，估算出了不同物种群 及物种间的分化时间(图2), 重建了巨蚓科物种祖先 分布区域。研究结果表明, 巨蚓科所在的巨蚓超科 与正蚓超科分化时间大约在侏罗纪早期，这一时间 点与Bouché (1998)估计的巨蚓超科的起源时间(约 220 百万年)相近，而在这个时期冈瓦纳大陆还未发 生分裂。地球生物在经历二叠纪一三叠纪大灭绝事 件与三叠纪一侏罗纪大灭绝事件后, 虾蚓祖先分化 出巨蚓超科、正蚓超科与沙蚓超科等，巨蚓超科发 生后向亚洲板块与澳洲板块扩散，又分化为了巨蚂 科、棘蚓科、八毛蚓科(Octochaetidae)、真蚓科和寒 宪蚓科(Ocnerodrilinae)等5科(Qiu \& Bouché, 1998)。 在亚洲板块与澳洲板块发展的巨蚓科于白严纪后 期出现科内的分化，而中国巨蚓科远盲蚓属与腔蚂 属主要类群的祖先大约起源于晚白严纪末期到新 生代初期, 约65.32 Ma (58.89-72.89 Ma)。根据分化 时间估计及祖先分布重建可知，中国巨蚓科蚯蚓主 要类群在晚白严纪末期主要分布于云南与广西(图 3), 晚白严纪末期到新生代初期主要类群由云南与 广西向北、向东等方向扩散开来, 故进一步推测出 中国巨蚓科虾蚓主要类群的祖先起源于中南半岛。

由于蚯蚓的演化是受多种因素(如基因、古地 理和生态因素)相互作用影响而形成的(Wares \& Turner, 2003; Byrne et al, 2008), 现存物种的分布格 局往往是祖先起源后，经扩散与隔离分化在时空尺 度上交替发生的综合作用所造就的(Keppel et al, 2009)。根据祖先分布区域重建结果，再结合相关地 


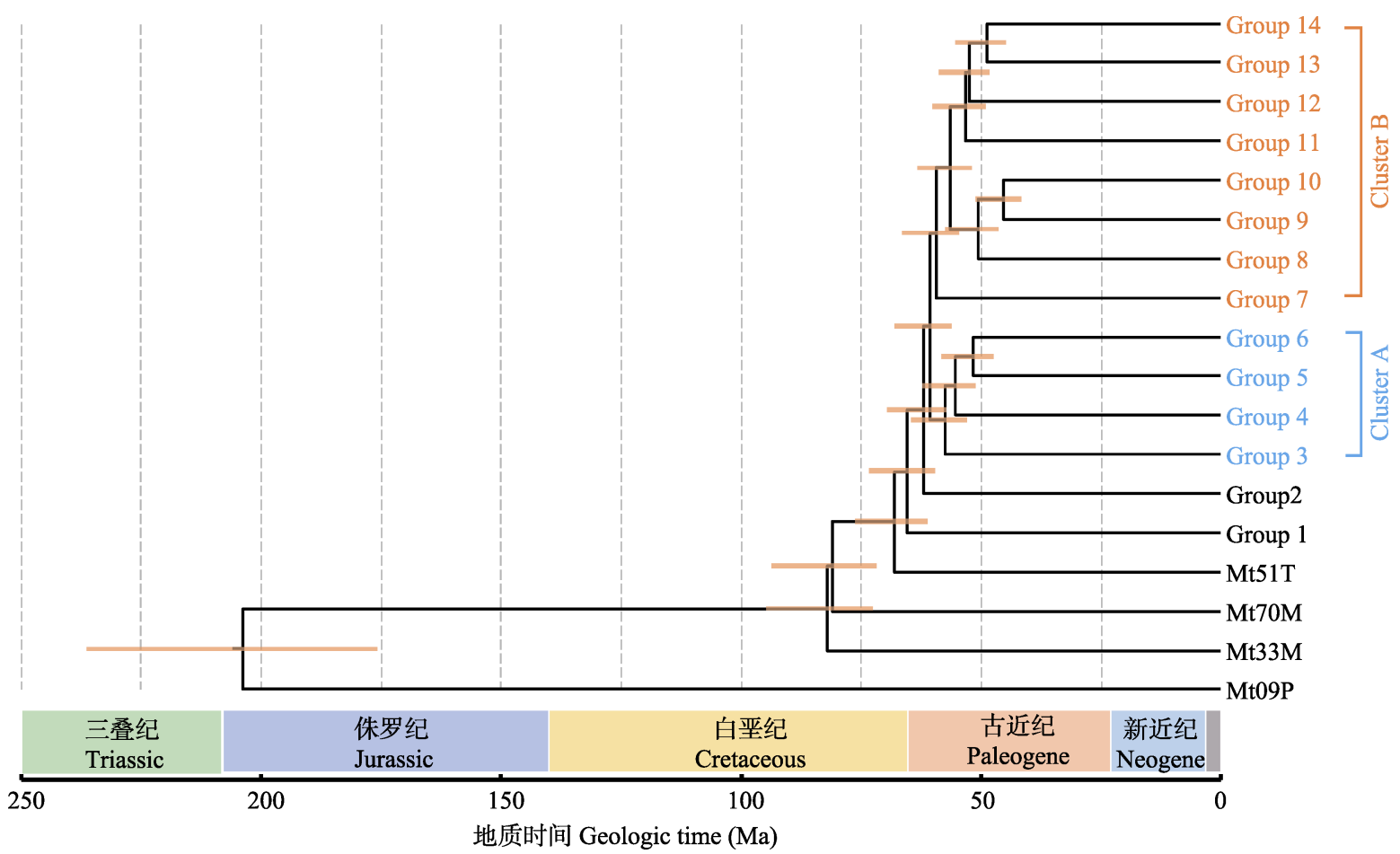

图2 中国巨蚓科蚯蚓主要类群的分化时间。Mt09P为外群, Mt33M为环棘蚓属物种, Mt70M与 Mt51T为2个远盲蚓属物种。 Fig. 2 Divergence times of major groups belong to the family Megascolecidae from China. Mt09P, Outgroup; Mt33M, Belonging to genus Perionyx; Mt70M and Mt51T, Belonging to genus Amynthas.

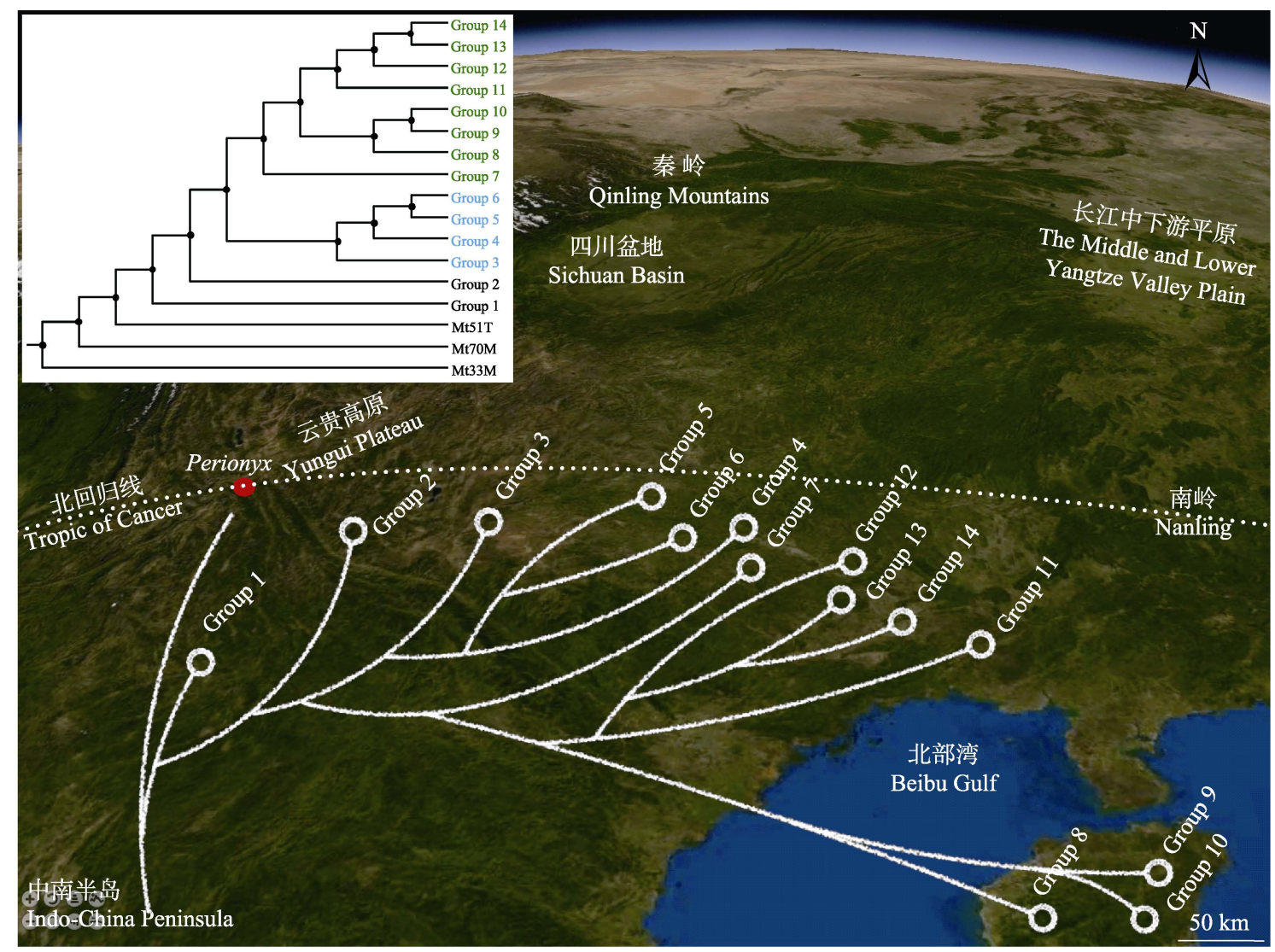

图3 中国巨蚓科蚯蚓分子系统发育树在地图上的投影

Fig. 3 Projection map of phylogenetic tree of the family Megascolecidae from China 
质变迁资料, 可在物种阶元上探讨我国巨蚓科蛏蚓 主要类群的分化历程与物种的扩散过程。中国巨蚓 科祖先于中南半岛进入中国后, 分化为 14 个主要的 进化类群(图2), 这些进化类群主要在大陆内向北、 向东扩散，逐步占据适合生存的环境，而第8、9、 10进化类群的祖先进入海南后主要在岛内分化与 扩散(图3; 蒋际宝, 2016)。中国巨蚓科主要类群在 新生代时期物种数目大幅增加; 到上新世以后物种 增加速率大幅下降(图4), 其原因可能在于第四纪冰 期造成了一定数量物种的灭绝, 也有可能是由于区 域环境容纳量趋于饱和, 物种扩散速率变缓。中国 北方地区的蚯蚓物种很少, 且多是广布物种, 推测 其原有的蝶蚓物种多数已在第四纪冰期灭绝, 仅有 少数子遗物种得以生存, 现有广布物种多是冰期后 南方种群向北扩散分布的结果。

现有的多项研究表明, 岛屿虾蚓的祖先往往来 自大陆。海南岛巨蚂科祖先大约是始新世初期(约 $50.60 \mathrm{Ma}$ )从大陆扩散到现在海南岛的南部, 随后主 要在岛内分化扩散, 少数物种的祖先又在始新世中 期(约42.13 Ma)从海南岛经陆桥扩散到大陆(Zhao, 2012; 赵琦, 2015; 蒋际宝, 2016); 这一结果与植物 区系研究的结果相似, Zhu (2016)根据植物学研究 提出, 海南岛在始新世时(约53 Ma)连接着越南和 我国广西, 后来发生了向东南的移动和旋转; 在更 新世时期，海南岛与大陆之间进行了不少物种传播, 因为这个时期琼州海峡的陆桥又断断续续地出现。 赵琦通过估算海南岛物种分化时间, 并结合海南岛

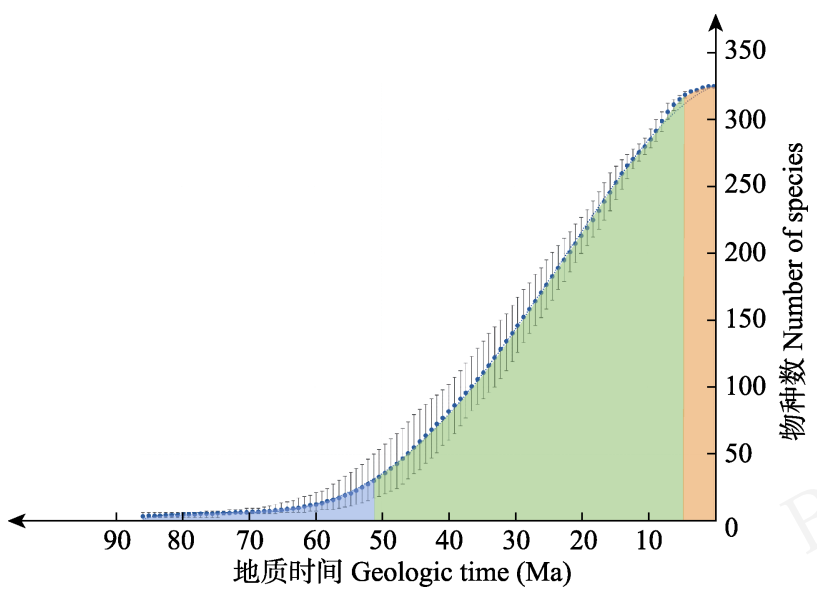

图4 中国巨蚓科蚯蚂物种分化数随时间的变化

Fig. 4 Diversification of Megascolecidae species from China through time
古生物地理学资料重建虹蚓扩散历程，可发现岛内 巨蚓科蚟蚓约在新生代中期经历了 3 次主要的物种 分化时期，这与古地理历史资料基本吻合(Zhao, 2012; 赵琦, 2015)。Tsai等也多次指出台湾岛上巨蚓 科蚯蚓的祖先主要来自于大陆东南部，台湾海峡的 陆桥在更新世的周期性出现, 为台湾岛和大陆的一 些物种提供了隔离演化的机会(Tsai et al, 2000a, b; Shen et al, 2002)。Chang和Chen (2005)采用线粒体 COI基因论证了台湾岛内福尔摩沙腔蚓(Metaphire formosae) 和友学腔蚓(Metaphire yuhsii)姊妹物种的 生物地理历史假说，初步估计二者的分化时间距今 2.1-2.5 Ma，这与台湾西部麓山隆起后山川、河流的 形成时间相符。Chang等(2008)又基于线粒体COI、16S 和 ND1 基因分析了台湾岛内腔蚂属formosae-group 的13个物种的种群遗传变异, 指出该类群受台湾岛 周边地区的多重分散隔离影响，并初步估计各个物 种发生异域分化时间为2.7-5.8 Ma，略早于晚中新 世时期的台湾岛迅速提升阶段(2.5-5.0 Ma), 物种 进入岛屿后种群扩散至整个岛屿。

从时间尺度上来看，中国巨蚓科物种的分化多 发生于10 Ma之前，一千万年内发生的分化节点仅 占总数的 $14 \%$, 最短的物种形成年限也需 4.31 百万 年。由此可见, 虹蚓的物种形成时间较长, 可能与 其较弱的扩散能力有关(Mazaud \& Bouché, 1980)。

综上所述，巨蚓科虾蚓于晚白严纪末期至新生 代初期进入中国，逐渐扩散开来，并适应多样的生 境, 主要在新生代演化出繁多的新物种, 形成现有 的分布格局。已有研究基于形态研究与系统发育分 析展示了中国蚳蚓起源、分化与扩散历程, 但还未 更系统地结合地理格局、地质历史及环境因子定量 分析类群演化与古地理、生物与非生物因素间的关 系，造成中国巨蚓科蛏蚓的进化机制还不明确。

\section{中国巨蚂科的形态特征演化}

䗒蚓从水中向陆地的进化过程中，挖掘洞穴的 需要促进了其个体增大、前部隔膜增厚、体壁肌肉 组织形成及消化器官的改变, 进而影响了这些器官 临近体节腔室中性器官的发展(Omodeo, 2000)。到 了陆栖生活阶段，各科虾蚓为适应不同的环境压 力，也演化出了多样的外部与内部形态特征。

中国巨蚓科虾蚓主要器官的演化得到了多次 
探讨。王海军(2005)硕士论文 ${ }^{(1)}$ 采用因子对应分析方 法对我国已记录的188种远盲蚓属和腔蚓属蚟蚓的 形态学数据开展多元统计分析, 在属、物种阶元探 讨了其形态学系统发育和结构-功能关系, 研究认 为受精囊孔的特征是远盲蚓属和腔蚓属的重要特 征, 盲肠也有较重要的意义。有关海南岛环毛类(我 国巨蚓科蚯蚓除掘穴环棘蚓(Perionyx excavatus)外, 其余均属于环毛类) 41 个物种(亚种)的分子系统发 育关系研究也发现, 在其进化过程中, 与性选择相 关的形态特征最为重要, 与环境选择相关的形态特 征次之(Zhao, 2012; 赵琦, 2015)。下文对部分重要 特征取得的研究进展进行论述:

(1)受精囊孔演化。孙静(2017)针对我国远盲蚓 属55个物种(亚种)开展分子分析与祖先特征重建并 重点研究了受精囊孔特征的演化, 发现远盲蚓属代 表性物种被分成了两个明显的地理分支, 分支内物 种往往具有不同的受精囊孔对数与位置, 而具有相 同受精囊孔特征的后代不一定位于同一单系群内 (附录4)。由于受精囊孔对数或位置不是共同起源的, 其演化是拥有流体静力骨骼(hydrostatic skeleton)的 虹蚓为适应生存环境而选择增加或减少生殖投入 的策略(Christensen, 1984, 1994; Qiu et al, 1998), 造 成同一进化支上不同物种为适应所处的生存环境 可演化出不同的受精囊孔对数与位置, 故受精囊孔 特征不应作为单一的分类标准。同时，无受精囊的 孤雌生殖物种并未单独聚成一支, 而是分散到了不 同的进化支中，与相邻进化支的非孤雌生殖物种存 在很多相似特征, 据此也可推测无受精囊的孤雌生 殖物种是由近缘的非孤雌生殖物种退化生殖器官 而成(蒋际宝, 2016)。此外, 研究过程中还发现有些 物种正在向孤䧳生殖方向发展, 其种群常呈现出过 渡态特征。有些非孤雌生殖的广布物种, 例如皮质 远盲蚂(Amynthas corticis), 部分种群出现了无受精 囊现象; 或者少数孤雌生殖广布物种, 例如珠串远 盲蚂(Amynthas moniliatus), 部分种群却具有完整的 受精囊。总体而言, 受精囊孔对数的演化较为复杂。 Bouché和Qiu (1998)认为受精囊孔向前和向后延伸, 与运动功能及其他器官发展等相关, 如体腔张力、 砂囊、肌肉质体节或环带的增长或缩短等(Bouché, 1998; Qiu \& Bouché, 1998), 但具体演化模式至今

(1) 王海军 (2005) 中国环毛类蝶蚓分类学和系统发育研究. 硕士学位 论文, 上海交通大学, 上海.
也不明确。

(2)盲肠特征演化。Sims和Easton (1972)通过形 态数值分析认为盲肠特征是巨蚓科环毛类蛲蚓近 缘属的重要区分特征。巨蚓科蚯蚓的盲肠有单式指 状、过渡态与复式3种形状，盲端朝向前方，为重要 的消化腺体(陈义, 1956)。通过对巨蚓科祖先的盲肠 特征开展分析，可知中国巨蚓科蚯蚓祖先的盲肠形 态是单式的, 各个进化类群的祖先也多有单式盲 肠。该特征在巨蚓科演化过程中整体呈现从单式向 过渡态或复式演化，但在有些单系群中会出现祖征 重现现象(evolutionary reversal) (蒋际宝, 2016)。

(3)祖先特征重建。基于已构建的多基因分子系 统发育树开展祖先特征重建分析, 也可推测出中国 巨蚓科虾蚓祖先的形态。蒋际宝(2016)的研究显示 巨蚓科祖先中等体长，体节数约为60-120, 背侧具 有色素, 刚毛数适中(40-60根), 第一背孔位于 $9 / 10$ 节间或之后，环带占满3节，受精囊孔3对位于6/7到 8/9节间, 雄孔位于体表中等突起上, 受精囊孔间距 或雄孔间距介于 $1 / 4$ 到 $1 / 2$ 节周长，内部8/9到 $9 / 10$ 节 间隔膜缺失，砂囊占据2节，单式盲肠起于XXVII节， 精巢囊、储精囊、前列腺及受精囊均发达。由此推 测，这些出现在晚白严纪末期至新生代初期的特征 使得巨蚓科祖先可较好地适应中国南方当时已逐 步稳定的地质条件，并伴随着新生代时期被子植物 的繁盛得以极大地发展, 再缓慢地扩散到湿热条件 适宜的不同区域，进而形成丰富的物种多样性。

值得注意的是，进化树上主要类群的各个形态 特征, 特别是生殖特征, 存在着连续而复杂的演化, 暂时无法用统一的特征划分标准来区分。而现阶段 通用的环毛类虾蚓分类系统是Sims和Easton (1972) 建立的, 他们试图通过数值分类的方法解决巨蚂科 这一主要类群的系统发育问题, 但最终只是在其论 著中将环毛属(Pheretima)重新分成了数个独立的属, 并将各属大量物种按照受精囊孔的对数和位置、雄 孔的位置以及是否全雄等特征在属以下区分为不 同的物种群(species-group)。这项工作减轻了聚类物 种的难度, 但并不能明确地阐释属、种阶元的系统 发育关系, 进而阐明环毛类蚯蚓的形态演化, 因此 其属、物种群阶元的划分受到了普遍质疑，有待新 分类系统的推出进行改进(James, 2005; Zhao, 2012; 孙静, 2013; 蒋际宝, 2016)。 


\section{3 研究展望}

近年来, 针对地球板块运动、地质变迁、气候 变化等因素与蚟蚓类群分化的相互关系, 及其对类 群遗传结构的影响和地理分布格局形成等科学问 题的研究, 已成为当前探索蚯蚓起源与演化机制的 热点(Bouché, 1998; Omodeo, 2000; Domínguez et al, 2015)。为更好地阐述中国巨蚓科蚯蚓的起源与演化, 未来研究可在获取中国、日本、韩国以及东南亚、 澳洲等地巨蚓科样本和更多分子标记信息的基础 上, 结合古地理变迁、气候变化以及植物区系的相 关资料, 全面地分析巨蚓科蛙蚓属、种与种群的演 化机制。以往的研究探讨了我国巨蚓科蚳蚓主要类 群的起源中心, 也推测了分化时间及扩散历程, 但 具体的扩散方向与速率、演化机制及影响因素等仍 不明确。而蝶蚓的演化和发展是伴随着地球的古地 理学变迁(大陆板块漂移)和陆地高等植物的演化和 发展而进行的(邱江平, 2000)。因此，可将系统发育 分析与地理格局、地质历史及环境因子相结合, 并 评估出各个参数、因素在种群或其他类群的时空分 布格局形成中的作用, 定量分析类群演化与古地 理、生物与非生物因素间的关系, 进而全面厘清巨 蚓科䗒蚓演化的机制。同样地, 也可根据蚯蚓演化 不同阶段类群分子和形态特征的变化, 寻找出相关 环境因子或生物因素, 进而验证或推测所处环境的 地质变迁、气候变化及植物协同演化过程。

同时, 可结合正蚓科、链胃蚓科等其他科综合 探讨蚯蚓的进化模式。从总体进化历程上看, 中国 巨蚓科蚳蚓的演化时间不长, 新生代及以后时期独 特的地理稳定性为中国巨蚓科演化出丰富的物种 多样性创造了客观条件(蒋际宝, 2016)。而链胃蚓科 主要分布在缅甸、印度尼西亚、印度与我国南方等 地, 与巨蚓科虾蚓都具有印度马来区的特点, 可能 有相似的起源和物种分化扩散历程(陈义, 1956)。正 蚂科起源于晚白严纪的古北区欧洲板块, 在我国分 布的物种可能多为外来种(Qiu \& Bouché, 1998; Domínguez et al, 2015)。因此, 系统采集我国这两个 较广泛分布的科, 与巨蚓科一起探讨演化历程, 将 有助于理解相关类群的起源、分化与扩散, 为系统 地认识整个虹蚓的进化奠定坚实基础。

\section{参考文献}

Bouché MB (1972) Lombriciens de France: Écologie et
Systématique. Institut National de la Recherche Agronomique, Paris.

Bouché MB (1983) The establishment of earthworm communities. In: Earthworm Ecology (ed. Satchell JE), pp. 431-448. Springer Netherlands, Dordrecht.

Bouché MB (1998) L'éolution spatiotemporelle des lombrixiens. Documents Pédozoologiques et Intégrologiques, 3, 1-28.

Byrne M, Yeates DK, Joseph L, Kearney M, Bowler J, Williams MAJ, Cooper S, Donnellan SC, Keogh JS, Leys R (2008) Birth of a biome: Insights into the assembly and maintenance of the Australian arid zone biota. Molecular Ecology, 17, 4398-4417.

Chang CH, Chen JH (2005) Taxonomic status and intraspecific phylogeography of two sibling species of Metaphire (Oligochaeta: Megascolecidae) in Taiwan. Pedobiologia, 49, 591-600.

Chang CH, Lin SM, Chen JH (2008) Molecular systematics and phylogeography of the gigantic earthworms of the Metaphire formosae species group (Clitellata, Megascolecidae). Molecular Phylogenetics and Evolution, 49, 958-968.

Chen Y (1956) Earthworms in China. Science Press, Beijing. (in Chinese) [陈义 (1956) 中国蝶蚂. 科学出版社, 北京.]

Christensen B (1984) Asexual propagation and reproductive strategies in aquatic Oligochaeta. Hydrobiologia, 115, 91-95.

Christensen B (1994) Annelida-Clitellata. In: Reproductive Biology of Invertebrates, Vol. VI, Part B (eds Adiyodi KG, Adiyodi RG), pp. 1-23. John Wiley \& Sons, Hoboken.

Csuzdi C (2012) Earthworm species, a searchable database. Opuscula Zoologica, 43, 97-99.

Domínguez J, Aira M, Breinholt JW, Stojanovic M, James SW, Pérez-Losada M (2015) Underground evolution: New roots for the old tree of lumbricid earthworms. Molecular Phylogenetics and Evolution, 83, 7-19.

Gates GE (1972) Burmese earthworms: An introduction to the systematics and biology of megadrile oligochaetes with special reference to Southeast Asia. Transactions of the American Philosophical Society, 62, 1-326.

Huang J, Xu Q, Sun ZJ, Wang C, Zheng DM (2006) Research on earthworm resources of China. I. Checklist and distribution. Journal of China Agricultural University, 11, 9-20. (in Chinese with English abstract) [黄健, 徐芹, 孙振钧, 王冲, 郑东梅 (2006) 中国蛅蚂资源研究. I. 名录及分布. 中国 农业大学学报, 11, 9-20.]

James SW (2005) Preliminary molecular phylogeny in the Pheretima group of genera (Crassiclitellata: Megascolecidae) using Bayesian analysis. In: Advances in Earthworm Taxonomy II (Annelida: Oligochaeta) (eds Pop VV, Pop AA), pp. 129-142. Cluj University Press, Cluj-Napoca.

Jamieson BGM (1988) On the phylogeny and higher classification of the Oligochaeta. Cladistics, 4, 367-401.

Jiang JB (2016) Taxonomy and Molecular Phylogeny of the Family Megascolecidae Earthworms from China. PhD dis- 
sertation, Shanghai Jiao Tong University, Shanghai. (in Chinese with English abstract) [蒋际宝 (2016) 中国巨蚓 科蚯蚓分类与分子系统发育研究. 博士学位论文, 上海 交通大学, 上海.]

Keppel G, Lowe AJ, Possingham HP (2009) Changing perspectives on the biogeography of the tropical South Pacific: Influences of dispersal, vicariance and extinction. Journal of Biogeography, 36, 1035-1054.

Mazaud D, Bouché MB (1980) Introduction en surpopulation et migrations de lombriciens marqués. In: Soil Biology as Related to Land Use Practices (ed. Dindal DL), pp. 687-701. EPA, Washington, DC.

Michaelsen W (1900) Oligochaeta. R. Friedländer und Sohn, Berlin.

Michaelsen W (1921) Neue und wenig bekannte Oligochäten aus skandinavischen Sammlungen. Arkiv för Zoologi, 13, 1-25.

Michaelsen W (1929a) Zur Stammesgeschichte der Oligochäten. Zwiss Zoology, 134, 693-716.

Michaelsen W (1929b) The Oligochaete fauna of China. Lingnan Science Journal, 8, 157-166.

Michaelsen W (1931) The Oligochaeta of China. Peking Natural History Bulletin, 5, 1-24.

Omodeo P (1998) History of Clitellata. Italian Journal of Zoology, 65, 51-73.

Pérez-Losada M, Breinholt JW, Porto PG, Aira M, Domínguez J (2011) An earthworm riddle: Systematics and phylogeography of the Spanish Lumbricid Postandrilus. PLoS ONE, 6, e28153.

Qiu JP, Bouché MB (1998) Révision des taxons supraspécifiques de Lumbricoidea. Documents Pédozoologiques et Intégrologiques, 3, 179-216.

Qiu JP, Bouché MB, Patricio S (1998) Gathering, rationalization and managment of knowledge applied to the earthworm biodiversity. Documents Pédozoologiques et Intégrologiques, 3, 57-118.

Qiu JP (1999) Earthworms and their application in environment protection. I. Earthworms and their functions in ecosystem. Journal of Shanghai Agricultural College, 17, 227-232. (in Chinese with English abstract) [邱江平 (1999) 蚯蚓及其 在环境保护上的应用. I. 蛏蚓及其在自然生态系统中的 作用. 上海农学院学报, 17, 227-232.]

Qiu JP (2000) Earthworms and environmental protection. Guizhou Science, 18, 116-133. (in Chinese with English abstract) [邱江平 (2000) 蚯蚓与环境保护. 贵州科学, 18, 116-133.]

Shen HP, Tsai CF, Tsai SC (2002) Description of a new earthworm belonging to the genus Amynthas (Oligochaeta: Megascolecidae) from Taiwan and its infraspecific variation in relation to elevation. Raffles Bulletin of Zoology, 50, 1-8.

Shen HP, Chang CH, Chih WJ (2016) Four new earthworm species of the genus Amynthas (Megascolecidae: Oligochaeta) from southwestern Taiwan with re-description of tungpuensis Tsai, Shen and Tsai, 1999. Journal of Natural History, 50, 1889-1910.

Sims RW, Easton EG (1972) A numerical revision of the earthworm genus Pheretima auct. (Megascolecidae: Oligochaeta) with the recognition of new genera and an appendix on the earthworms collected by the Royal Society North Borneo Expedition. Biological Journal of the Linnean Society, 4, 169-268.

Sun J (2013) Taxonomy and Molecular Phylogeny of Amynthas Earthworms from China. PhD dissertation, Shanghai Jiao Tong University, Shanghai. (in Chinese with English abstract) [孙静 (2013) 中国远盲属蚳蚓分类学及分子系统 发育研究. 博士学位论文, 上海交通大学, 上海.]

Sun J, James SW, Jiang JB, Yao B, Zhang LL, Liu M, Qiu JP, Hu F (2017) Phylogenetic evaluation of Amynthas earthworms from South China reveals the initial ancestral state of spermathecae. Molecular Phylogenetics and Evolution, 115, 106-114.

Tsai CF, Shen HP, Tsai SC (2000a) Native and exotic species of terrestrial earthworms (Oligochaeta) in Taiwan with reference to Northeast Asia. Zoological Studies, 39, 285-294.

Tsai CF, Tsai SC, Liaw GJ (2000b) Two new species of protandric pheretimoid earthworms belonging to the genus Metaphire (Megascolecidae: Oligochaeta) from Taiwan. Journal of Natural History, 34, 1731-1741.

Wares JP, Turner TF (2003) Phylogeography and diversification in aquatic mollusks. In: Molecular Systematics and Phylogeography of Mollusks (eds Lydeard C, Lindberg D), pp. 229-269. Smithsonian Books, Washington, DC.

Zhang WX, Hendrix PF, Dame LE, Burke RA, Wu J, Neher DA, Li JX, Fu SL (2013) Earthworms facilitate carbon sequestration through unequal amplification of carbon stabilization compared with mineralization. Nature Communications, 4, 2576.

Zhao Q (2012) Taxonomy, Phylogeny and Paleogeography of Pheretimoid Earthworm Species (Megascolecidae: Amynthas, Metaphire) in Hainan Island (China). PhD dissertation, University of Rennes 1, Rennes.

Zhao Q (2015) Taxonomy, Phylogeny and Paleogeography of Pheretimoid Earthworm Species in Hainan Island (China). PhD dissertation, Shanghai Jiao Tong University, Shanghai. (in Chinese with English abstract) [赵琦 (2015) 中国海南 岛环毛类蚯蚓分类学、系统发育学和古生物地理学研究. 博士学位论文, 上海交通大学, 上海.]

Zhao Q, Zhang M, Dong Y, Qiu JP (2017) New species of Megascolecidae (Oligochaeta) from Hainan Island, China. Annales Zoologici, 67, 221-227.

Zhu H (2016) Biogeographical evidences help revealing the origin of Hainan Island. PLoS ONE, 11, e0151941.

(责任编委: 傅声雷 责任编辑: 黄祥忠、周玉荣) 


\section{附录 Supplementary Material}

附录1 早三叠纪时期蚯蚓各超科在盘古大陆的分布(译自Bouché, 1983)

Appendix 1 Distribution of earthworm superfamilies in relation to Pangaea in the early Triassic (220 Ma) (adapted from Bouché, 1983)

http://www.biodiversity-science.net/fileup/PDF/2018105-1.pdf

\section{附录2 白严纪早期(120 Ma), 与劳亚大陆从冈瓦纳大陆分离事件相关的蚯蚓各科的分布(译自Bouché, 1983)}

Appendix 2 Distribution of families in relation to the separation of Laurasia from Gondwana in the early Cretaceous (120 Ma) (adapted from Bouché, 1983)

http://www.biodiversity-science.net/fileup/PDF/2018105-2.pdf

附录3 根据形态特征演化推断的寡毛纲系统发育树(译自Bouché, 1998)

Appendix 3 Evolution phylogenetic tree of Oligochaeta inferred from morphological characteristics (adapted from Bouché, 1998) http://www.biodiversity-science.net/fileup/PDF/2018105-3.pdf

\section{附录4 中国远盲蚓属56种蚯蚓受精囊孔祖先特征重建(Sun et al, 2017)}

Appendix 4 Ancestral character state reconstructions of spermathecal pores in 56 Amynthas species on the Bayesian tree (adapted from Sun et al, 2017)

http://www.biodiversity-science.net/fileup/PDF/2018105-4.pdf 


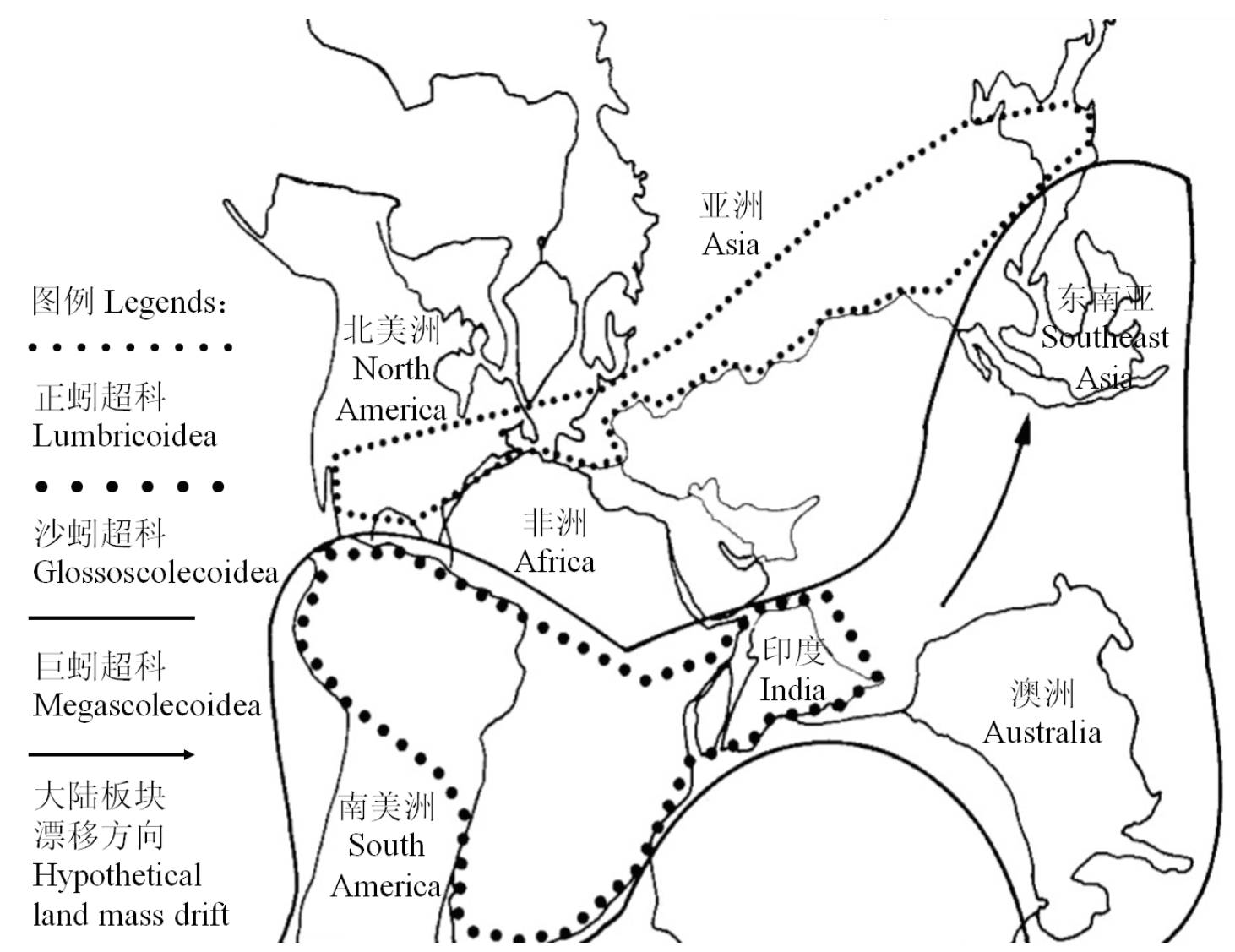

附录1 早三叠纪时期蛲蚂各超科在盘古大陆的分布 (译自Bouché, 1983)

Appendix 1 Distribution of earthworm superfamilies in relation to Pangaea in the early Triassic (220 Ma) (adapted from Bouché, 1983) 


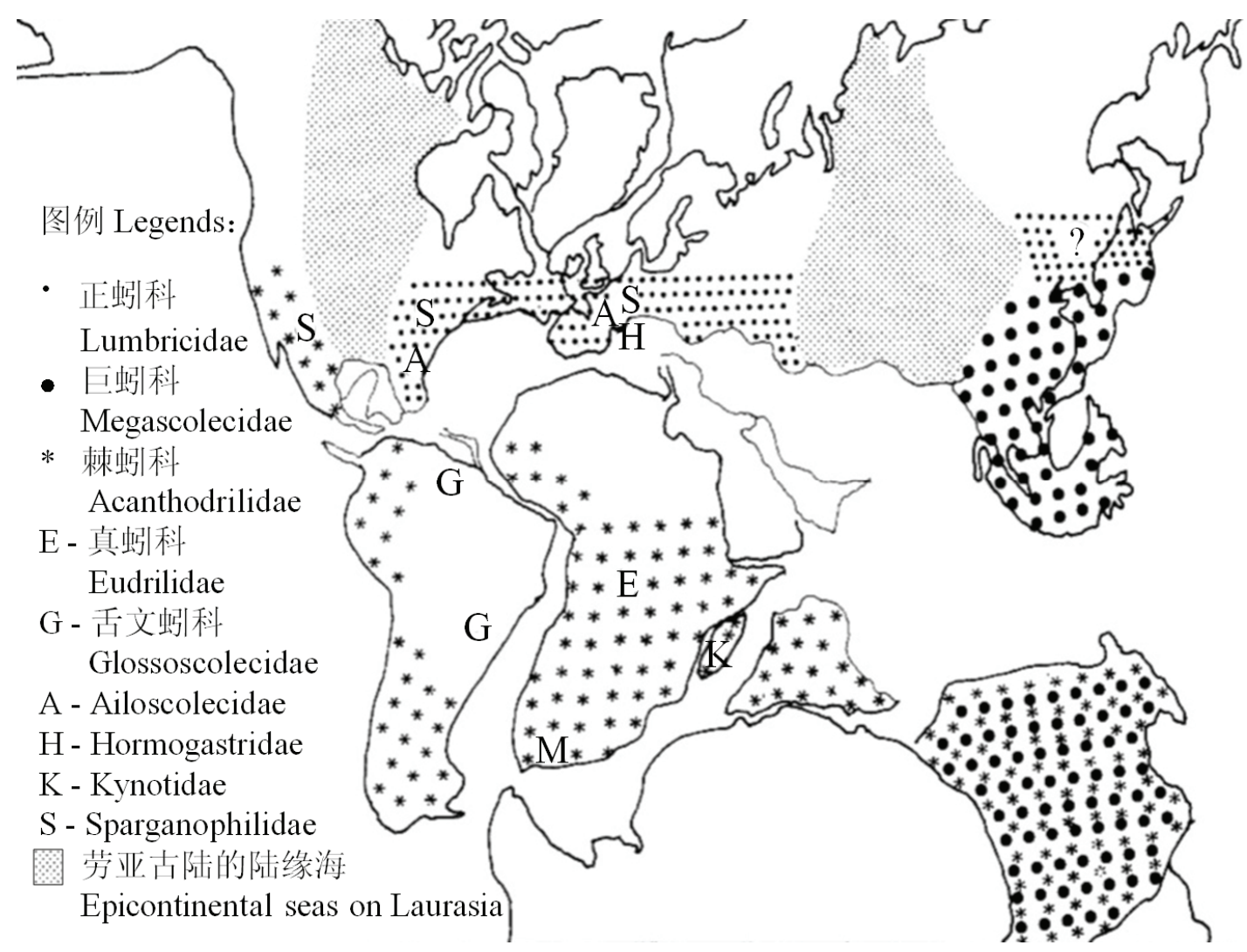

附录2 白严纪早期(120 Ma), 与劳亚大陆从冈瓦纳大陆分离事件相关的蚯蚓各科的分布(译自Bouché, 1983)

Appendix 2 Distribution of families in relation to the separation of Laurasia from Gondwana in the early Cretaceous (120 Ma) (adapted from Bouché, 1983) 


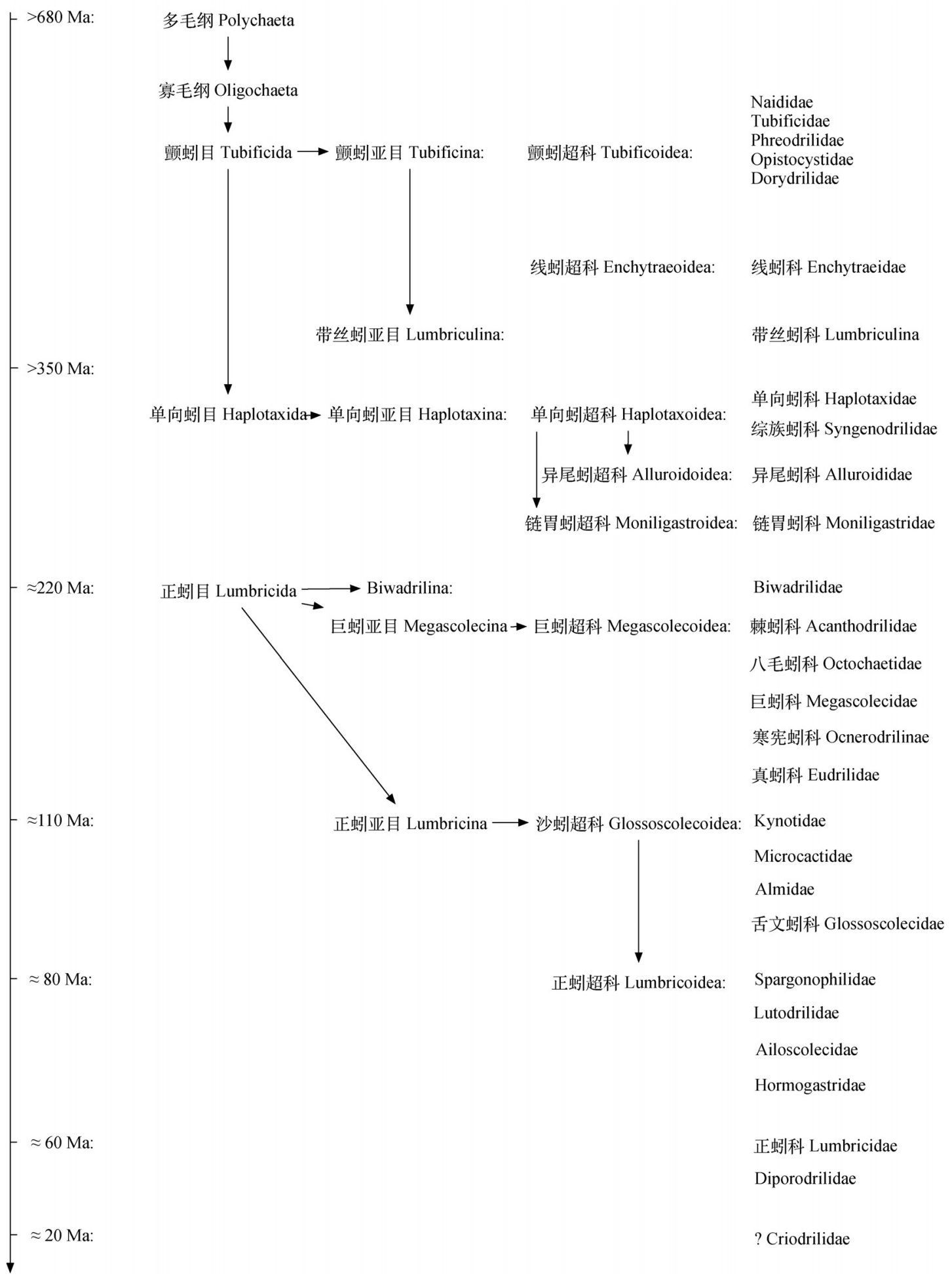

附录3 根据形态特征演化推断的寡毛纲系统发育树(译自Bouché, 1998)

Appendix 3 Evolution phylogenetic tree of Oligochaeta inferred from morphological characteristics (adapted from Bouché, 1998) 


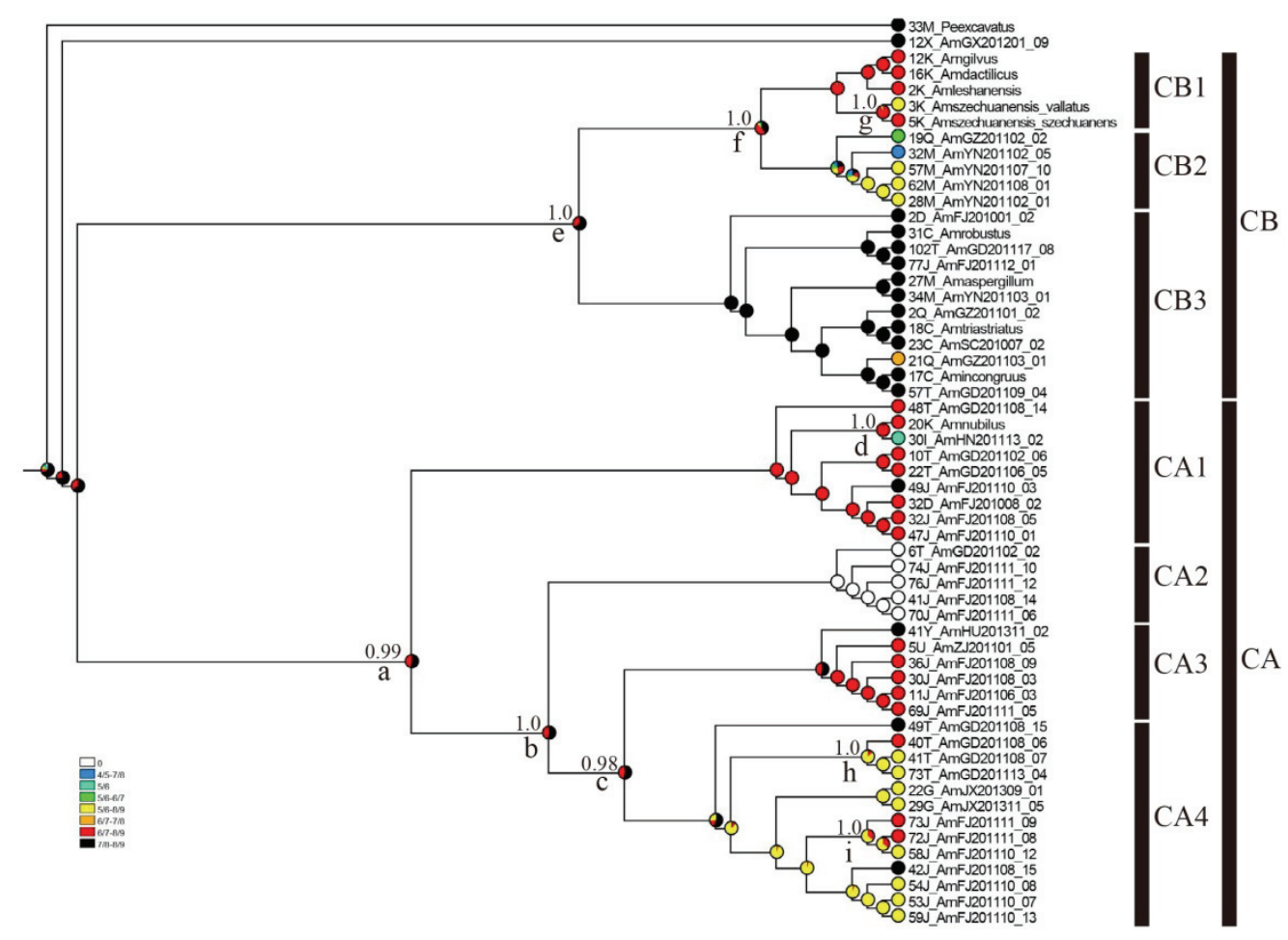

附录4 中国远盲蚓属56种蚯蚓受精囊孔祖先特征重建(Sun et al, 2017)

Appendix 4 Ancestral character state reconstructions of spermathecal pores in 56 Amynthas species on the Bayesian tree (adapted from Sun et al, 2017) 